\title{
Prioritizing the Patient Perspective in Oncologic Surgery
}

\author{
Nikhil Panda, MD ${ }^{1,3}$, and Alex B. Haynes, MD, MPH ${ }^{2,3}$ \\ ${ }^{1}$ Department of Surgery, Massachusetts General Hospital, Boston, MA; ${ }^{2}$ Department of Surgery and Perioperative Care, \\ Dell Medical School, The University of Texas at Austin, Austin, TX; ${ }^{3}$ Safe Surgery Program, Ariadne Labs, Boston, MA
}

Meaningfully defining the successful delivery of cancer care relies not only on traditional oncologic outcome measures, but also the patient's perspective as captured through patient-reported outcome measures (PROMs). In this issue of Annals of Surgical Oncology, three educational reviews have discussed the role of PROMs in measuring the quality of cancer care delivery, cancerspecific instruments, as well as challenges around the collection and integration of PROMs into daily clinical practice. $^{1-3}$

There has been an evolution of the quality metrics for oncologic surgery. Dr. Billig and colleagues describe how metrics that were historically used to benchmark surgical quality and effectiveness were dominated by clinical outcome measures, such as morbidity, mortality, or readmission rates. ${ }^{3}$ The same was true in oncologic surgery, with additional clinical metrics like resection margins or quality of lymphadenectomy. In response to national initiatives advocating for value- rather than volume-based care, there has been a growing emphasis on incorporating PROMs alongside these measures.

The result is a shift in how the healthcare system and associated stakeholders think of quality. Quality is not defined by the absence of unexpected or undesired healthcare events, but rather as a health state described by patients and defined in accordance with their goals and values. When programs have incorporated PROMs into quality measurement, there have been improvements in patient satisfaction, enhanced communication among

(C) Society of Surgical Oncology 2019

First Received: 6 August 2019;

Published Online: 26 August 2019

A. B. Haynes, MD, MPH

e-mail: alex.haynes@austin.utexas.edu patients and their care teams, reduction in resource utilization, and observed survival benefits. ${ }^{4}$ Based on this, reimbursement models have gradually begun to incentivize hospital systems to assess performance as measured in part by these patient-centered outcomes.

There are several important considerations healthcare systems must appreciate when designing programs to integrate PROMs into clinical care. These range from careful selection of generic, disease- and/or cancer-specific instruments, timing and frequency of survey administration, and incorporating results to improve care. These are summarized clearly by Dr. Ivaturi and colleagues' educational review. ${ }^{1}$ However, there are two considerations that warrant additional emphasis: the resources necessary to implement PROMs, and the culture around these measures.

Creating and maintaining a PROM initiative requires substantial human and financial resources. As described by Dr. Phillips and colleagues, initiatives like the PatientReported Outcomes Measurement Information System (PROMIS) sponsored by the National Institute of Health have provided a valid library of instruments for point of care use. ${ }^{2}$ While many of these contemporary instruments are open source, other validated surveys and scoring systems remain proprietary. The method and frequency of survey administration may require developing or licensing existing platforms, representing a substantial initial investment and additional resources for maintenance. There are also costs involved with scaling these initiatives, from ensuring instruments are available in multiple patient languages to integrating PROM data into multiple electronic health records. Finally, there is associated time, effort, and opportunity costs for providers. PROMs, fundamentally, are additional patient data collected for clinical use and quality assessment. Gathering, processing, regulating, and interpreting these data, without an appreciation of their potential value, often becomes an added responsibility for providers. 
These challenges have led to innovative research focused on novel methods for capturing PROMs. For example, in an attempt to limit the burden of multiple surveys, there is ongoing psychometric research and incorporation of methods like computer adaptive testing to minimize the requisite time and items to assess quality of life. ${ }^{5}$ Regarding timing and frequency of collection, there is growing evidence originating in the field of clinical psychology to support the frequent assessment of patients in their natural environments to obtain more accurate assessments of quality of life and minimize bias. ${ }^{6}$ There is also an increasing role of technology to support the delivery, completion, and scoring of surveys, which may lead to more efficient clinical intervention. This has significant potential in oncologic surgery in particular, where improvements in perioperative care and standardized recovery pathways have led to a growing number of patients recovering outside of a monitored clinical setting.

There is also an opportunity to harness technology to develop new patient-centered outcome measures. Our research team is investigating how passive data, such as smartphone sensor and usage data, may provide insights into postoperative quality of life. ${ }^{7,8}$ For example, postoperative movement and physical activity may be captured by smartphone accelerometer and GPS sensors; fine motor control may be measured in part by the smartphone gyroscope; and social activity may be manifested in communication logs. Combining these types of passive data with PROMs collected remotely through patient smartphones yield momentary descriptions of quality of life across physical and psychosocial domains. Ideally, these types of data could be used in near real time to detect changes in quality of life throughout each phase of cancer treatment.

The success of incorporating any form of patient-centered outcome measures will depend ultimately on understanding the culture around these data. Traditional outcome measures have had systems developed that integrate these data into clinical care and surveillance. The interpretation of these data is relatively straightforward for clinicians. PROMs and other novel patient-centered metrics, despite being quantitative measures, describe health states as domains rather than absolute values and may be more challenging to translate into clinical action. Clinicians who are unfamiliar with PROMs are unlikely to buyinto new initiatives without education, training, and appreciation of their value. Likewise, health systems cannot assume that patients recognize the purpose and potential value of providing self-reported quality of life data. Few patients are given opportunities to appreciate how their data are being used to direct their care. As such, patients may be hesitant or even unwilling to provide responses to surveys. There is a need for further mixedmethod research involving patients and clinicians to further understand these nuances of PROMs.

In summary, when successfully incorporated into patient care, PROMs have clearly emerged as a valuable quality measure in oncologic surgery. The challenges facing scaling these initiatives, namely resource limitations and culture, represent an opportunity for clinicians, patients, and policy leaders to pursue innovative research and support implementation. Progress in these areas will translate into advances in oncologic surgery toward true patientcentered care.

\section{REFERENCES}

1. Ivatury S, Hazard-Jenkins H, Brooks G, McCleary N, Wong S, Schrag D. Translation of patient-reported outcomes in oncology clinical trials to everyday practice. Ann Surg Oncol. 2019. https://d oi.org/10.1245/s10434-019-07749-2.

2. Phillips J, Wong S. Patient-reported outcomes in surgical oncology: an overview of instruments and scores. Ann Surg Oncol. 2019. https://doi.org/10.1245/s10434-019-07752-7.

3. Billig J, Sears E, Travis B, Waljee J. Patient-reported outcomes: understanding surgical efficacy and quality from the patient's perspective. Ann Surg Oncol. 2019. https://doi.org/10.1245/s1043 4-019-07748-3.

4. Basch E, Deal AM, Kris MG, Scher HI, Hudis CA, Sabbatini P, et al. Symptom monitoring with patient-reported outcomes during routine cancer treatment: a randomized controlled trial. J Clin Oncol. 2016;34(6):557-65. https://doi.org/10.1200/jco.2015.63. 0830 .

5. Gibbons C, Bower P, Lovell K, Valderas J, Skevington S. Electronic quality of life assessment using computer-adaptive testing. J Med Internet Res. 2016;18(9):e240.

6. Shiffman S, Stone AA, Hufford MR. Ecological momentary assessment. Annu Rev Clin Psychol. 2008;4:1-32.

7. Torous J, Kiang MV, Lorme J, Onnela J-P. New tools for new research in psychiatry: a scalable and customizable platform to empower data driven smartphone research. JMIR Ment Health. 2016;3(2):e16.

8. Panda N, Solsky I, Haynes AB. Redefining shared decisionmaking in the digital era. Eur J Surg Oncol. 2019. https://doi.org/ 10.1016/j.ejso.2019.07.025.

Publisher's Note Springer Nature remains neutral with regard to jurisdictional claims in published maps and institutional affiliations. 